\title{
Application of Decision Support System for Agro Technology Transfer (DSSAT) to Simulate Agronomic Practices for Cultivation of Maize in Southern Highland of Tanzania
}

\author{
Lusajo Henry Mfwango*, Sangharsh Kumar Tripathi, Gogumalla Pranuthi, \\ Sunil Kumar Dubey, Vijay Kumar Gubey \\ Department of Water Resources and Irrigation Engineering, Water Institute, Dar es Salaam, Tanzania \\ Email: *lusajojo2002@yahoo.com
}

How to cite this paper: Mfwango, L.H., Tripathi, S.K., Pranuthi, G., Dubey, S.K. and Gubey, V.K. (2018) Application of Decision Support System for Agro Technology Transfer (DSSAT) to Simulate Agronomic Practices for Cultivation of Maize in Southern Highland of Tanzania. Agricultural Sciences, 9, 910-923.

https://doi.org/10.4236/as.2018.97063

Received: June 26, 2018

Accepted: July 27, 2018

Published: July 30, 2018

Copyright $\odot 2018$ by authors and Scientific Research Publishing Inc. This work is licensed under the Creative Commons Attribution International License (CC BY 4.0).

http://creativecommons.org/licenses/by/4.0/

\begin{abstract}
The southern highlands zone of Tanzania is the one of the most potential area for agriculture contributes up to $46 \%$ of the total country's maize production. However, the rate of maize production tends to decrease with time due of poor agronomic practices. The aim of this study was to simulate the effect of nitrogen dose and plant spacing on grain yields from five selected maize varieties. Decision Support System for Agrotechnology transfer crop model was used for this purpose. Based on the agroecological zones, six sites were selected which includes Ihumbu farm, Mwazye and Nyera Estate Mbozi, Lupa Tinga Tinga, Santilya and Mbinga. Maize varieties H614, Kitumani Composite I, H511, H626 and H612; Spacing $(90 \times 30 \mathrm{~cm}$ and $60 \times 30 \mathrm{~cm})$ and nitrogen dose $(0,50,100,150$ and $200 \mathrm{~kg} / \mathrm{ha})$ were simulated. It was found that only H614 (4610.9 kg/ha) and Kitumani Composite I (3998.7 kg/ha) maize varieties performed well at the spacing of $60 \times 30 \mathrm{~cm}$ and up to the nitrogen dose of $150 \mathrm{~kg} / \mathrm{ha}$. Therefore the two maize varieties H614 and Kitumani Composite I could be recommended for cultivation at the spacing of $60 \times 30$ $\mathrm{cm}$ and nitrogen dose of $150 \mathrm{~kg} / \mathrm{ha}$ for improving production of maize in southern highland of Tanzania.
\end{abstract}

\section{Keywords}

Southern Highlands (Tanzania), DSSAT, Nitrogen Dose, Plant Spacing and Maize Varieties

\section{Introduction}

The economy of Tanzania is highly dependent on agriculture which provides the 
source of livelihood for over $80 \%$ of the population. The agricultural sector constitutes nearly $24 \%$ of the value of the national income (GDP) [1]. To a large extent the sector is characterized by traditional production systems which rely on indigenous varieties whose overall productivity is generally low.

The southern highlands zone of Tanzania contributes about $46 \%$ of national maize production and it accounts for nearly $90 \%$ of the maize purchased for the National Food Security Granary [2]. This zone comprises of four regions viz. Iringa, Mbeya, Rukwa, and Ruvuma. Over $80 \%$ of the maize produced in this region is grown by smallholders under a wide range of agronomic practices, climatic conditions, and socioeconomic conditions. Beside the contribution of the zone to the country maize production, still there is a large gap between national average yield ( $1.4 \mathrm{t} / \mathrm{ha})$ and potential yields $(7.0 \mathrm{t} / \mathrm{ha})$ [3]. Among the agronomic factors responsible for low yield of the region which are plant population in the field, application of inappropriate amount of inorganic fertilizers and use of low yielding varieties/cultivars are of prime importance [4]. Crop row spacing is another important agronomic management strategy used by farmers to optimize the husbandry of the soil and plant ecosystem from sowing to harvest with the goal of increasing the production of crops [5]. Row spacing has a special significance since it is ultimately related with plant population, root development, plant growth and fruiting [6]. Maize is well known for its high demand of nutrients. In addition to other agronomic practices, fertilization may be the most important way to maintain high crop productivity and soil fertility [7]. The fact that nitrogen has a good effect on plant productivity is universally accepted. Nevertheless, it also has a pollutant effect on the environment when dissolved and leach down to ground water/aquifer zones when applied irrationally. When nitrogen is rapidly leached down to ground water, they affect human and animal health [8]. Therefore, judicious use of mineral nitrogen fertilizer should be promoted on improvement maize productivity [9], without reduction in yields and much adverse impact on environment (soil and ground water quality). The development of appropriate management practices can be achieved by employing crop models like DSSAT in simulation studies.

The decision support system for agro technology transfer (DSSAT) crop model has been used for different applications in various countries around the world. It was originally developed by an international network of scientists, cooperating in the International Benchmark Sites Network for Agrotechnology Transfer project [10], to facilitate the application of crop system models such as Crop Environment Resource Synthesis (CERES) maize model in a systems approach to agronomic research. Its initial development was motivated by a need to integrate knowledge about soil, climate, crops, and management for making better decisions about transferring production technology from one location to others where soils and climate differed [11]. The DSSAT helps decision-makers by reducing the time and human resources required for analyzing complex alternative decisions [12]. It also provides a framework for scientific cooperation through 
research to integrate new knowledge and apply it to research questions. The objective of this study is to simulate the effect of nitrogen dose, spacing and suitability of varieties on increasing grain yield of maize grown in southern highland zone of Tanzania by application of CERES-maize model of DSSAT.

\section{Materials and Methods}

\subsection{Description of the Study Area}

The southern highlands zone found on the latitude of $7^{\circ}$ and $11.5^{\circ} \mathrm{S}$ and longitudes of $30^{\circ}$ and $38^{\circ} \mathrm{E}$ (Figure 1). The zone occupies an area of $250,000 \mathrm{~km}^{2}$ which is about $28 \%$ of the mainland area of Tanzania. The elevation from the sea level ranges from 400 and $3000 \mathrm{~m}$. The region experience semi-arid condition in some parts of Iringa region to high rainfall in highland areas (more than 2500 $\mathrm{mm}$ of rain per year) with cool temperatures. The rainfall pattern is unimodal with a long rainy season $(700 \mathrm{~mm}$ to over $2600 \mathrm{~mm}$ ) from November to May with a dry and cool spell between June and September [13]. The soil has low fertility, highly weathered and frequently acidic. Normally, maize is planted between the ends of November and early of December and harvested between April and July, depending on the weather and the variety grown [14]. For the purposes of the study six (6) sites were bee selected, Ihumbu farm $(-7.88,35.8)$, Mwazye $(-8.43,31.71)$, Nyera Estate Mbozi $(-9.16,33.11)$, Santilya $(-9.08,33)$, Lupa Tinga Tinga $(-8.01,33.26)$ and Mbinga $(-10.91,35.0)$.

\subsection{Model Input Data}

DSSAT crop simulation models in generally predict crop yield as a function of

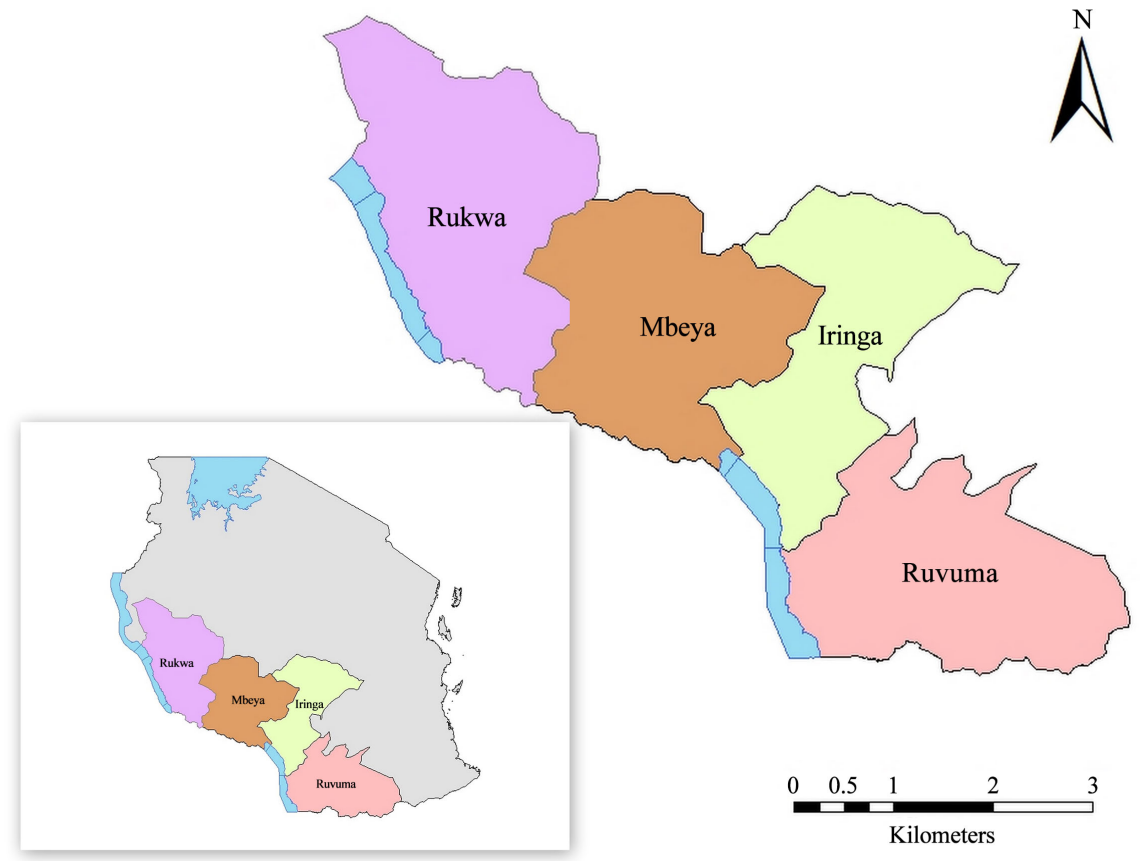

Figure 1. Map of Tanzania showing the location of Southern Highland zone. 
weather conditions, soil conditions and crop management practices. The minimum daily weather data required running the DSSAT models which includes maximum and minimum temperature $\left({ }^{\circ} \mathrm{C}\right)$, rainfall $(\mathrm{mm})$, and sunshine hours (hours/day) which were downloaded from the New_LocClim of Food and Agriculture Organisation. The New_locClim (an abbreviation for "Local Climate"), a software program and database, provides estimates of average climatic conditions at locations for which no observations are available. The sunshine hours (hours/day) were converted into solar radiation $\left(\mathrm{MJm}^{-2} \mathrm{day}^{-1}\right)$ by using Weatherman tool of DSSAT.

Soil characteristics data which includes clay fraction (\%), silt fraction (\%), stones fraction (\%), organic carbon (\%), CEC (Cation Exchange Capacity) (cmol/kg), pH (in water), horizon thickness (depth), surface characteristics such as soil color, Land slope, permeability, drainage class, and soil series name. These data were obtained from HWSD (Harmonized World Soil Database) which was created by the Food and Agriculture Organization of the United Nations and the International Institute for Applied Systems Analysis (IIASA) [15]. These organizations took the initiative of combining the recently collected vast volumes of regional and national updates of soil information with the information already contained within the 1:5,000,000 scales FAO-UNESCO Digital Soil Map of the World, into a new comprehensive HWSD.

The crop management data were obtained from the survey report, documented by [14]. These data includes crop cultivar, planting date, seedling rate (plant/hill), plant spacing $(\mathrm{cm})$, row spacing $(\mathrm{cm})$, and planting depth $(\mathrm{cm})$. Others include fertilizer application (dates, amounts, type of material, and method of application), tillage/intercultural operations (dates, depth equipment used), organic fertilizer amendments (date, amount, type and method of application). The collected soil and weather data were for running the model were compiled and presented as shown in Table 1 and Figure 2 respectively.

\subsection{Simulation Processing}

The CERES-Maize model incorporated in DSSAT v4.5 was used for simulation. Simulation was done separately for each selected sites in all treatment combination (cultivar, spacing and nitrogen dose) making 75 runs. Cultivars more or less cultivated/available in that region are used in the simulation to determine their suitability to that particular region. For this purpose five varieties of maize were selected Kitumani Composite 1 (V1), H614 (V2), H626 (V3) H612 (V4) and H511 (V5). Planting date was set on 20 November where by Dry seed of Maize at the depth of $5 \mathrm{~cm}$ where planted at seed rate of 2 plants/hill. Spacing treatments used in the simulation are $S 1(90 \times 30 \mathrm{~cm}), \mathrm{S} 2(60 \times 30 \mathrm{~cm})$ and $\mathrm{S} 3(90 \times 50 \mathrm{~cm})$. The number of plants at seedling and emergency by calculation was set 8 plants per $\mathrm{m}^{2}$ and 7 plants per $\mathrm{m}^{2}$ respectively at spacing one (S1) making the population of 70,000 plants per hectare. At spacing two (S2) and the same seed rate, the number plants at seedling was 12 plants per $\mathrm{m}^{2}$ and at emergency was 11 plants 

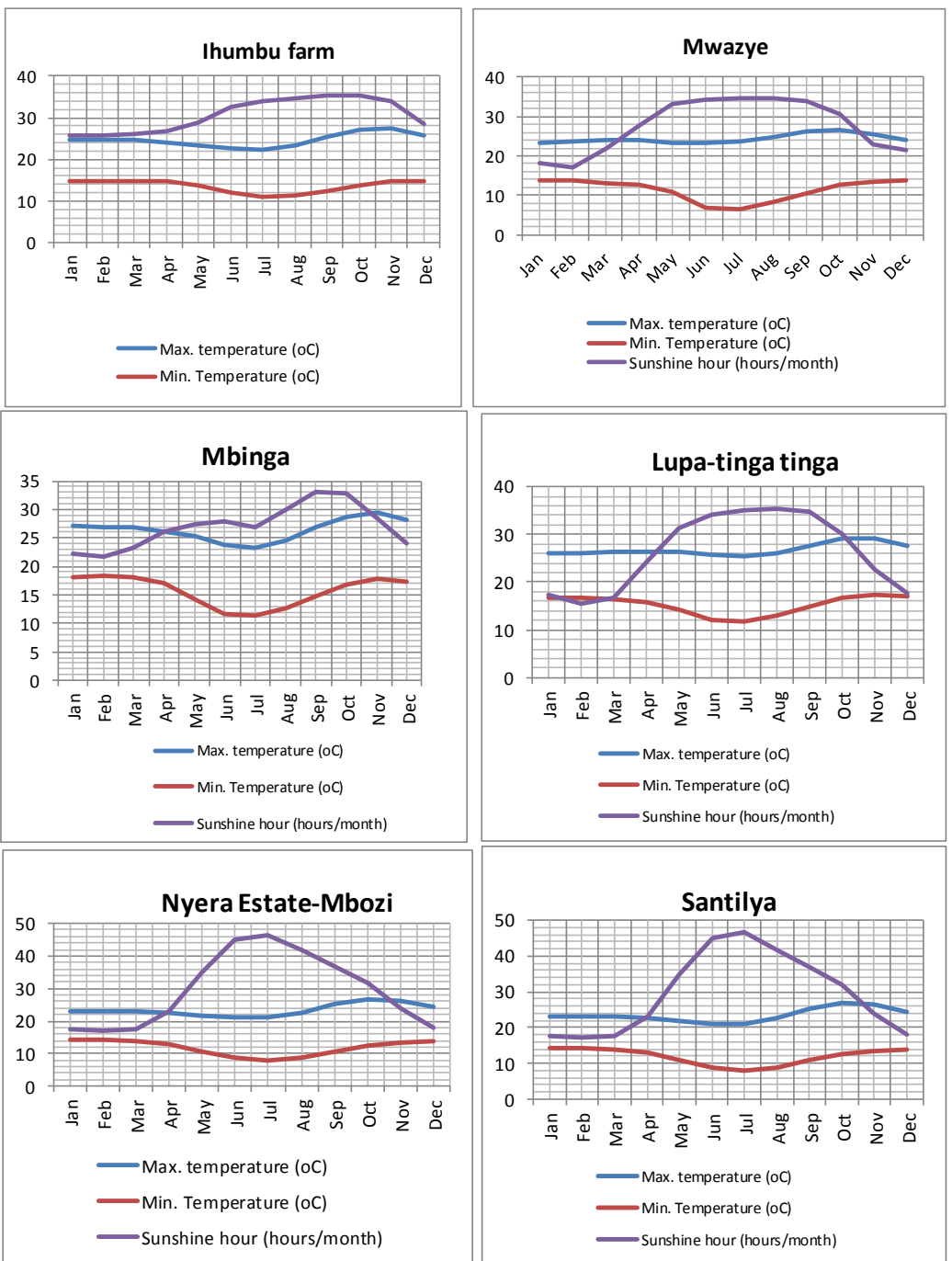

(a)

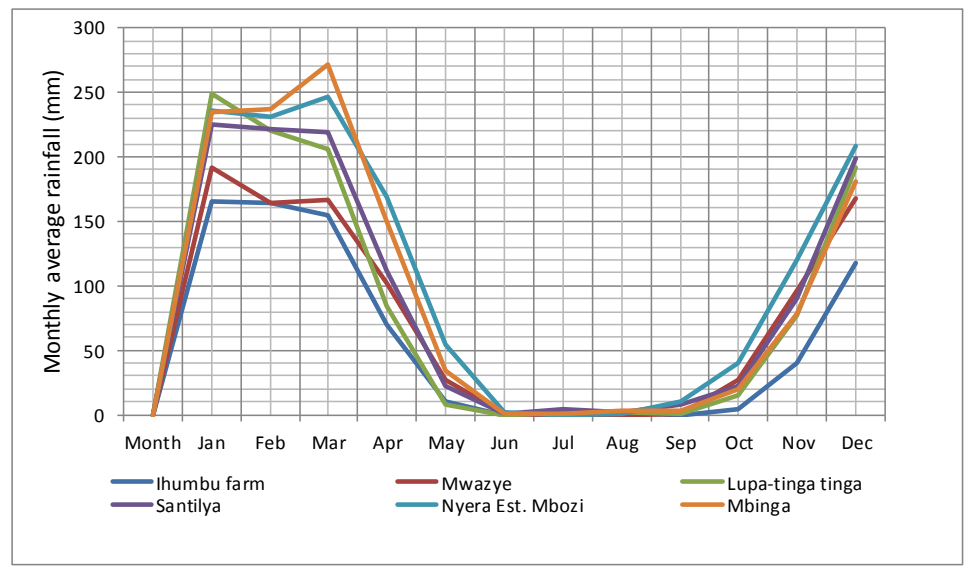

(b)

Figure 2. (a) A graph of average monthly minimum and maximum temperature and sunshine hours at the selected sites of Southern Highlands of Tanzania; (b) A graph of average monthly minimum and maximum temperature and sunshine hours at the selected sites of Southern Highlands of Tanzania. 
Table 1. Soil characteristics of different locations of Southern Highlands of Tanzania.

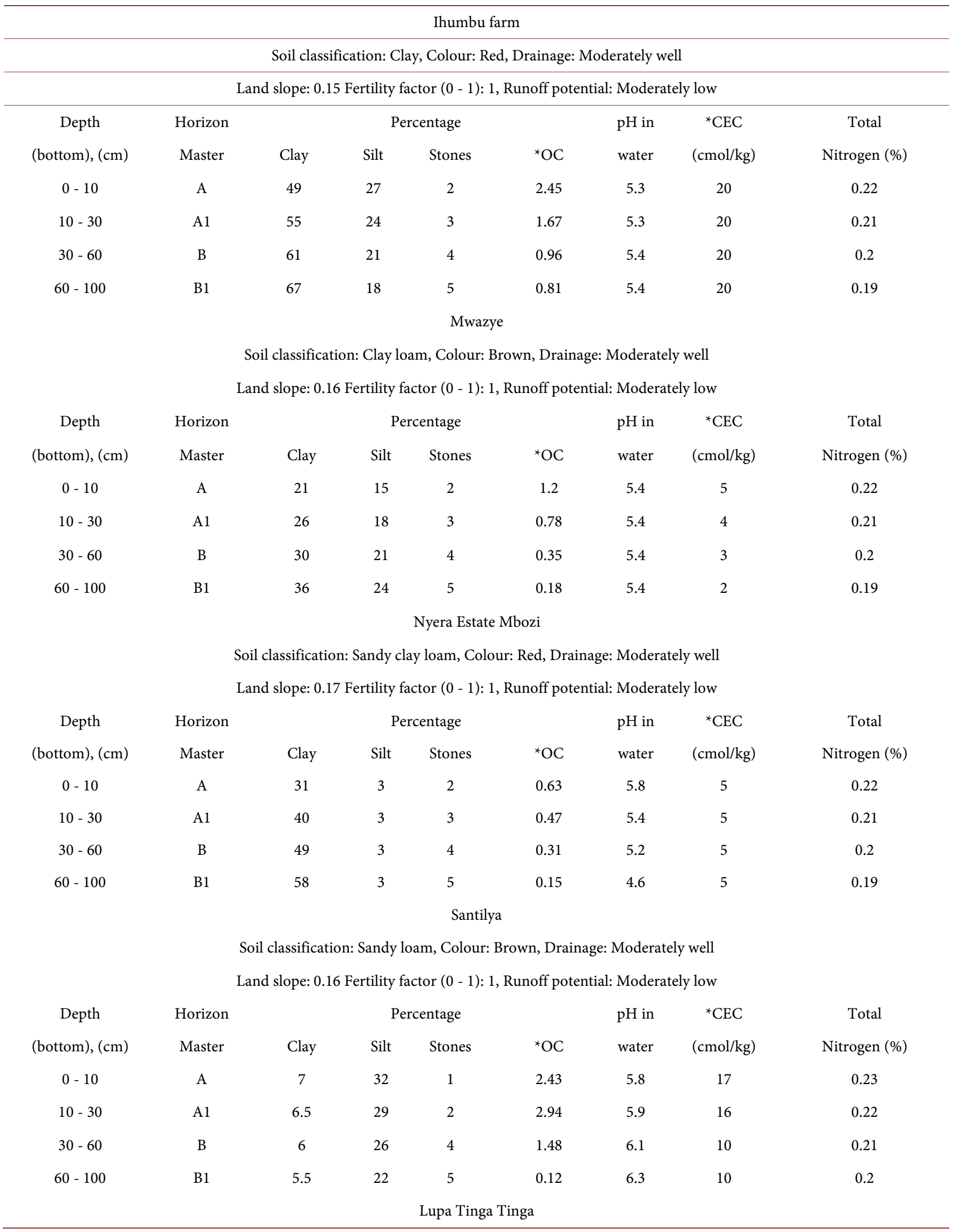


Soil classification: Sandy clay, Colour: Brown, Drainage: Moderately well

Land slope: 0.16 Fertility factor $(0-1): 1$, Runoff potential: Moderately low

\begin{tabular}{|c|c|c|c|c|c|c|c|c|}
\hline Depth & Horizon & & & entage & & $\mathrm{pH}$ in & ${ }^{\star} \mathrm{CEC}$ & Total \\
\hline (bottom), $(\mathrm{cm})$ & Master & Clay & Silt & Stones & ${ }^{*} \mathrm{OC}$ & water & $(\mathrm{cmol} / \mathrm{kg})$ & Nitrogen $(\%$ \\
\hline $0-10$ & A & 39 & 10 & 2 & 1.73 & 5.3 & 12 & 0.22 \\
\hline $10-30$ & $\mathrm{~A} 1$ & 42 & 9 & 3 & 1.26 & 5.4 & 10 & 0.21 \\
\hline $30-60$ & B & 44 & 8 & 4 & 0.78 & 5.6 & 9 & 0.2 \\
\hline $60-100$ & B1 & 46 & 6 & 5 & 0.31 & 5.8 & 7 & 0.19 \\
\hline
\end{tabular}

Soil classification: Sandy loam, Colour: Red, Drainage: Moderately well

Land slope: 0.16 Fertility factor (0 - 1): 1 , Runoff potential: Moderately low

\begin{tabular}{ccccccccc} 
Depth & Horizon & \multicolumn{2}{c}{ Percentage } & pH in & ${ }^{*} \mathrm{CEC}$ & Total \\
(bottom), (cm) & Master & Clay & Silt & Stones & ${ }^{*}$ OC & water & $(\mathrm{cmol} / \mathrm{kg})$ & Nitrogen (\%) \\
$0-10$ & A & 19 & 19 & 2 & 1.38 & 6.4 & 9 & 0.22 \\
$10-30$ & A1 & 26 & 18 & 3 & 0.91 & 5.7 & 8 & 0.21 \\
$30-60$ & B & 34 & 17 & 4 & 0.41 & 5 & 7 & 0.2 \\
$60-100$ & B1 & 41 & 15 & 5 & 0.21 & 4.3 & 6 & 0.19
\end{tabular}

${ }^{*} \mathrm{CEC}$ : Cation Exchange Capacity; ${ }^{\star} \mathrm{OC}$ : Organic Carbon.

per $\mathrm{m}^{2}$ which make the population of 110,000 plants per hectare. At spacing three (S3) and the same seed rate, the number plants at seedling was 4 plants per $\mathrm{m}^{2}$ and at emergency was 4 plants per $\mathrm{m}^{2}$ which make the population of 40,000 plants per hectare.

Varying nitrogen application from no nitrogen to $200 \mathrm{~kg}$ of nitrogen per hectare was used as treatments to study the effect of nitrogen on grain yields of maize for different cultivars. The nitrogen treatments used for yield simulation studies were N1 (0 kg/ha), N2 (50 kg/ha), N3 (100 kg/ha), N4 (150 kg/ha) and N5 $(200 \mathrm{~kg} / \mathrm{ha})$. Half of the total nitrogen is applied at the time of sowing; the remaining amount is given just before the juvenile stage of the crop. Urea is used as nitrogen supplement in this simulation study and the depth of placement is $5 \mathrm{~cm}$ during basal application and banded on the surface during the second application. Genetic coefficients of the maize varieties used in the simulation pre-existed within the model hence the model generated the data on the growth and developmental parameters without specifying it in the input data. The harvesting date is simulated for the crop when $50 \%$ of the plants reach harvest maturity (GS006).

\section{Results and Discussion}

The results of DSSAT simulated yield of maize obtained for six sites (Ihumbu 
farm, Mwazye and Nyera Estate Mbozi, Lupa Tinga Tinga, Santilya and Mbinga), five varieties (H614, Kitumani Composite I, H511, H626 and H612), five nitrogen doses $(0,50,100,150$ and $200 \mathrm{~kg} \mathrm{~N} / \mathrm{ha})$ and three spacings $(90 \times 30 \mathrm{~cm}$, $60 \times 30 \mathrm{~cm}$ and $90 \times 50 \mathrm{~cm}$ ) are presented in Table 2 and discussed in the forthcoming paragraphs.

\subsection{Response of Maize Varieties to Nitrogen with Respect to Grain Yield}

[16] explains that, an increase in grain yield of maize after application of Nitrogen is due to an increase in the number of ears per plant, increase in total dry matter distributed to the grain and increase in average ear weight. These plant characteristics tend to vary from one variety to another. The results from Table 2 show that, grain yield of all varieties tend to increase with the increasing rate of nitrogen, where by H614 variety followed by Kitumani composite I (KC I) respond faster than the other varieties (H612, H511 and H626). The overall mean grain yield in bracket of all varieties at spacing one (Sp 1) was H614 (4610.9 kg/ha), Kitumani Composite I (3998.7 kg/ha), H612 (2835.7 kg/ha), H511 (2231.6 kg/ha), and H626 (1673.3 kg/ha). The results are in agreement with [17] who found out positive response of different maize varieties on supply of nitrogen in increasing grain yield.

\subsection{Suitability of Maize Variety to Different Location}

Grain yield is the results of genetic potential and environmental interaction. From the results (Table 2), grain yield differ from one location to another at a given maize variety. An example of maize cultivar H614 at spacing one (S1) and $0 \mathrm{~kg} \mathrm{~N} / \mathrm{ha}$, maximum yield was observed at Santilya $(2684 \mathrm{~kg} / \mathrm{ha})$ and the minimum grain yield of $395 \mathrm{~kg} / \mathrm{ha}$ at Mbinga. The results are supported by [18] who report that maize varieties significantly differed in yield at different locations. [19] also reports the same on response to maize variety to different environmental condition. Overall results show that highest yield was obtained from H614 (4610.9 kg/ha) followed by Kitumani Composite I (3998.7 kg/ha), H612 (2835.7 kg/ha), H511 (2231.6 kg/ha) and H626 (1673.3 kg/ha) at spacing one (S1) and H614 (4724.5 kg/ha) followed by Kitumani Composite I (3465.3 kg/ha), H612 (2288.7 kg/ha), H511 (2151.6 kg/ha) and H626 (1626.9 kg/ha) at spacing 2 (S2).

\subsection{Effect of Plant Spacing on Grain Yield}

The effects of plant spacing on grain yield are also shown on Table 2. [20] has documented that, yield and yield component of corn are significantly affected by planting patterns, plant densities and maize hybrids. Corn hybrids respond differently to high plant density [21]. Plant density is a function of plant spacing on the field, the larger spacing results into low plant density per unit area. The overall results shows that the mean grain yield of all varieties was $3428.0 \mathrm{~kg} / \mathrm{ha}$ in spacing one (S1) and $3169.2 \mathrm{~kg} / \mathrm{ha}$ at spacing two (S2). Taking an example of 
Table 2. DSSAT forecasted grain yields of Maize (Zea mays L.) as affected by locations, variety, spacing and nitrogen dose in Southern Highlands of Tanzania.

\begin{tabular}{|c|c|c|c|c|c|c|c|c|c|c|c|c|c|c|c|c|c|}
\hline \multirow{3}{*}{ No. } & \multirow{3}{*}{ Treatments } & \multicolumn{16}{|c|}{ Location } \\
\hline & & \multicolumn{4}{|c|}{ Ihumbu } & \multicolumn{4}{|c|}{ Mwazye } & \multicolumn{4}{|c|}{ Nyera Estate Mbozi } & \multicolumn{4}{|c|}{ Santilya } \\
\hline & & S1 & S2 & S3 & Mean & S1 & S2 & S3 & Mean & S1 & S2 & S3 & Mean & S1 & S2 & S3 & Mean \\
\hline 1 & V1N1 & 1887.0 & 2058.0 & 1914.0 & 1953.0 & 634.0 & 576.0 & 793.0 & 667.7 & 459.0 & 449.0 & 491.0 & 466.3 & 2733.0 & 2976.0 & 2708.0 & 2805.7 \\
\hline 3 & V1N3 & 6200.0 & 7465.0 & 4412.0 & 6025.7 & 3769.0 & 4329.0 & 2286.0 & 3461.3 & 5140.0 & 4201.0 & 4309.0 & 4550.0 & 6080.0 & 6447.0 & 4504.0 & 5677.0 \\
\hline 4 & V1N4 & 6206.0 & 8114.0 & 4413.0 & 6244.3 & 2893.0 & 4959.0 & 2240.0 & 3364.0 & 6181.0 & 7229.0 & 4504.0 & 5971.3 & 6366.0 & 7642.0 & 4504.0 & 6170.7 \\
\hline \multirow[t]{2}{*}{5} & V1N5 & 6207.0 & 8117.0 & 4413.0 & 6245.7 & 2876.0 & 4092.0 & 2248.0 & 3072.0 & 6367.0 & 8216.0 & 4504.0 & 6362.3 & 6367.0 & 8199.0 & 4504.0 & 6356.7 \\
\hline & Mean & 5117.8 & 5918.0 & 3909.0 & 4981.6 & 2607.2 & 3327.0 & 2121.6 & 2685.3 & 4052.0 & 4454.0 & 3292.0 & 3932.7 & 5248.8 & 5934.8 & 4131.6 & 5105.1 \\
\hline 2 & $\mathrm{~V} 2 \mathrm{~N} 2$ & 3295.0 & 2388.0 & 4669.0 & 3450.7 & 1907.0 & 1738.0 & 2105.0 & 1916.7 & 1643.0 & 1362.0 & 2067.0 & 1690.7 & 4885.0 & 4845.0 & 4939.0 & 4889.7 \\
\hline 3 & V2N3 & 6590.0 & 4941.0 & 6134.0 & 5888.3 & 2846.0 & 2630.0 & 2930.0 & 2802.0 & 3935.0 & 3468.0 & 4665.0 & 4022.7 & 6437.0 & 6643.0 & 6235.0 & 6438.3 \\
\hline 4 & V2N4 & 8538.0 & 8416.0 & 7504.0 & 8152.7 & 3091.0 & 3361.0 & 3117.0 & 3189.7 & 6585.0 & 5236.0 & 6336.0 & 6052.3 & 7727.0 & 8177.0 & 7075.0 & 7659.7 \\
\hline \multirow[t]{2}{*}{5} & V2N5 & 9565.0 & 10284.0 & 7500.0 & 9116.3 & 3320.0 & 3562.0 & 3182.0 & 3354.7 & 8353.0 & 7892.0 & 7339.0 & 7861.3 & 8805.0 & 9481.0 & 7552.0 & 8612.7 \\
\hline & Mean & 5954.4 & 5519.2 & 5569.2 & 5680.9 & 2325.2 & 2343.4 & 2383.2 & 2350.6 & 4185.0 & 3672.8 & 4170.0 & 4009.3 & 6181.4 & 6366.0 & 5817.2 & 6121.5 \\
\hline 1 & V3N1 & 929.0 & 1069.0 & 851.0 & 949.7 & 467.0 & 443.0 & 457.0 & 455.7 & 405.0 & 415.0 & 436.0 & 418.7 & 1762.0 & 2008.0 & 1483.0 & 1751.0 \\
\hline 2 & V3N2 & 1066.0 & 1250.0 & 1580.0 & 1298.7 & 742.0 & 829.0 & 709.0 & 760.0 & 963.0 & 1095.0 & 905.0 & 987.7 & 2290.0 & 2429.0 & 2171.0 & 2296.7 \\
\hline 3 & V3N3 & 2262.0 & 1611.0 & 2643.0 & 2172.0 & 1106.0 & 1090.0 & 928.0 & 1041.3 & 1556.0 & 1552.0 & 1888.0 & 1665.3 & 2922.0 & 3064.0 & 2687.0 & 2891.0 \\
\hline \multirow[t]{2}{*}{5} & V3N5 & 3425.0 & 3489.0 & 2810.0 & 3241.3 & 1446.0 & 1469.0 & 1257.0 & 1390.7 & 3542.0 & 3079.0 & 3530.0 & 3383.7 & 3626.0 & 4181.0 & 3392.0 & 3733.0 \\
\hline & Mean & 2151.4 & 2066.4 & 2145.8 & 2121.2 & 997.2 & 1035.6 & 910.4 & 981.1 & 1781.4 & 1662.8 & 1964.0 & 1802.7 & 2783.2 & 3073.6 & 2566.8 & 2807.9 \\
\hline 1 & V4N1 & 1072.0 & 1123.0 & 1081.0 & 1092.0 & 455.0 & 382.0 & 464.0 & 433.7 & 375.0 & 377.0 & 407.0 & 386.3 & 1644.0 & 1725.0 & 1593.0 & 1654.0 \\
\hline 2 & V4N2 & 2447.0 & 1943.0 & 3109.0 & 2499.7 & 1337.0 & 1247.0 & 1416.0 & 1333.3 & 1042.0 & 1060.0 & 1303.0 & 1135.0 & 2511.0 & 2422.0 & 2672.0 & 2535.0 \\
\hline 3 & V4N3 & 4186.0 & 3736.0 & 3468.0 & 3796.7 & 2030.0 & 1937.0 & 1864.0 & 1943.7 & 2478.0 & 2020.0 & 3099.0 & 2532.3 & 3502.0 & 3361.0 & 3370.0 & 3411.0 \\
\hline 4 & V4N4 & 4515.0 & 5032.0 & 3468.0 & 4338.3 & 2262.0 & 2551.0 & 1540.0 & 2117.7 & 3970.0 & 3418.0 & 3535.0 & 3641.0 & 4065.0 & 4364.0 & 3528.0 & 3985.7 \\
\hline \multirow[t]{2}{*}{5} & V4N5 & 4495.0 & 5396.0 & 3468.0 & 4453.0 & 2130.0 & 2715.0 & 1445.0 & 2096.7 & 4602.0 & 4793.0 & 3535.0 & 4310.0 & 4404.0 & 4800.0 & 3536.0 & 4246.7 \\
\hline & Mean & 3343.0 & 3446.0 & 2918.8 & 3235.9 & 1642.8 & 1766.4 & 1345.8 & 1585.0 & 2493.4 & 2333.6 & 2375.8 & 2400.9 & 3225.2 & 3334.4 & 2939.8 & 3166.5 \\
\hline 1 & V5N1 & 1061.0 & 906.0 & 1793.0 & 1253.3 & 264.0 & 224.0 & 283.0 & 257.0 & 204.0 & 198.0 & 198.0 & 200.0 & 1854.0 & 1481.0 & 2342.0 & 1892.3 \\
\hline 2 & V5N2 & 4070.0 & 4430.0 & 2681.0 & 3727.0 & 1894.0 & 2868.0 & 1417.0 & 2059.7 & 2455.0 & 1516.0 & 2328.0 & 2099.7 & 3390.0 & 4258.0 & 2496.0 & 3381.3 \\
\hline 3 & V5N3 & 4078.0 & 5476.0 & 2655.0 & 4069.7 & 1798.0 & 2486.0 & 1394.0 & 1892.7 & 3591.0 & 4157.0 & 2567.0 & 3438.3 & 3594.0 & 4574.0 & 2567.0 & 3578.3 \\
\hline 4 & V5N4 & 4079.0 & 5478.0 & 2649.0 & 4068.7 & 1809.0 & 2496.0 & 1400.0 & 1901.7 & 3697.0 & 4998.0 & 2567.0 & 3754.0 & 3597.0 & 4722.0 & 2567.0 & 3628.7 \\
\hline \multirow[t]{2}{*}{5} & V5N5 & 4079.0 & 5479.0 & 2648.0 & 4068.7 & 1813.0 & 2500.0 & 1400.0 & 1904.3 & 3697.0 & 4998.0 & 2567.0 & 3754.0 & 3697.0 & 4861.0 & 2567.0 & 3708.3 \\
\hline & Mean & 3473.4 & 4353.8 & 2485.2 & 3437.5 & 1515.6 & 2114.8 & 1178.8 & 1603.1 & 2728.8 & 3173.4 & 2045.4 & 2649.2 & 3226.4 & 3979.2 & 2507.8 & 3237.8 \\
\hline 1 & N1 & 1346.6 & 1344.6 & 1535.6 & 1408.9 & 456.4 & 410.2 & 515.8 & 460.8 & 370.4 & 369.0 & 395.0 & 378.1 & 2209.2 & 2174.8 & 2282.2 & 2222.1 \\
\hline 2 & N2 & 3193.4 & 2769.4 & 3286.4 & 3083.1 & 1748.8 & 1872.2 & 1737.6 & 1786.2 & 1643.2 & 1441.6 & 1851.0 & 1645.3 & 3554.8 & 3672.8 & 3343.2 & 3523.6 \\
\hline
\end{tabular}


Continued

\begin{tabular}{|c|c|c|c|c|c|c|c|c|c|c|c|c|c|c|c|c|c|}
\hline 3 & N3 & 4663.2 & 4645.8 & 3862.4 & 4390.5 & 2309.8 & 2494.4 & 1880.4 & 2228.2 & 3340.0 & 3079.6 & 3305.6 & 3241.7 & 4507.0 & 4817.8 & 3872.6 & 4399.1 \\
\hline 4 & N4 & 5282.6 & 5990.6 & 4175.8 & 5149.7 & 2256.0 & 2942.8 & 1899.6 & 2366.1 & 4574.8 & 4610.8 & 4000.6 & 4395.4 & 5014.2 & 5718.2 & 4155.0 & 4962.5 \\
\hline 5 & N5 & 5554.2 & 6553.0 & 4167.8 & 5425.0 & 2317.0 & 2867.6 & 1906.4 & 2363.7 & 5312.2 & 5795.6 & 4295.0 & 5134.3 & 5379.8 & 6304.4 & 4310.2 & 5331.5 \\
\hline & Mean & 4008.0 & 4260.7 & 3405.6 & 3891.4 & 1817.6 & 2117.4 & 1588.0 & 1841.0 & 3048.1 & 3059.3 & 2769.4 & 2959.0 & 4133.0 & 4537.6 & 3592.6 & 4087.7 \\
\hline
\end{tabular}

\begin{tabular}{|c|c|c|c|c|c|c|c|c|c|c|c|c|c|}
\hline \multirow{3}{*}{ No. } & \multirow{3}{*}{ Treatments } & \multicolumn{11}{|c|}{ Locations } & \multirow{3}{*}{ Overall Mean } \\
\hline & & \multicolumn{4}{|c|}{ Lupa Tinga Tinga } & \multicolumn{4}{|c|}{ Mbinga } & \multicolumn{3}{|c|}{ Mean } & \\
\hline & & S1 & S2 & S3 & Mean & S1 & S2 & S3 & Mean & S1 & S2 & S3 & \\
\hline 1 & V1N1 & 1347.0 & 1564.0 & 1273.0 & 1394.7 & 471.0 & 440.0 & 503.0 & 471.3 & 1255.2 & 1343.8 & 1280.3 & 1293.1 \\
\hline 2 & V1N2 & 3529.0 & 3026.0 & 3667.0 & 3407.3 & 2329.0 & 1969.0 & 2488.0 & 2262.0 & 3437.0 & 3015.8 & 3446.5 & 3299.8 \\
\hline 3 & V1N3 & 5086.0 & 5703.0 & 3688.0 & 4825.7 & 4167.0 & 4240.0 & 2997.0 & 3801.3 & 5073.7 & 5397.5 & 3699.3 & 4723.5 \\
\hline 4 & V1N4 & 5086.0 & 6492.0 & 3688.0 & 5088.7 & 4495.0 & 5845.0 & 3162.0 & 4500.7 & 5204.5 & 6713.5 & 3751.8 & 5223.3 \\
\hline \multirow[t]{2}{*}{5} & V1N5 & 5086.0 & 6508.0 & 3688.0 & 5094.0 & 4559.0 & 5999.0 & 3209.0 & 4589.0 & 5243.7 & 6855.2 & 3761.0 & 5286.6 \\
\hline & Mean & 4026.8 & 4658.6 & 3200.8 & 3962.1 & 3204.2 & 3698.6 & 2471.8 & 3124.9 & 4042.8 & 4665.2 & 3187.8 & 3965.3 \\
\hline 1 & V2N1 & 1279.0 & 1213.0 & 1421.0 & 1304.3 & 414.0 & 395.0 & 456.0 & 421.7 & 1233.5 & 1115.2 & 1371.0 & 1239.9 \\
\hline 2 & V2N2 & 3243.0 & 2861.0 & 4308.0 & 3470.7 & 2038.0 & 1817.0 & 2688.0 & 2181.0 & 2835.2 & 2501.8 & 3462.7 & 2933.2 \\
\hline 3 & V2N3 & 6608.0 & 5270.0 & 6193.0 & 6023.7 & 4985.0 & 4982.0 & 4377.0 & 4781.3 & 5233.5 & 4655.7 & 5089.0 & 4992.7 \\
\hline 4 & V2N4 & 8143.0 & 8018.0 & 6855.0 & 7672.0 & 6206.0 & 7060.0 & 5581.0 & 6282.3 & 6715.0 & 6711.3 & 6078.0 & 6501.4 \\
\hline \multirow[t]{2}{*}{5} & V2N5 & 8357.0 & 8939.0 & 6855.0 & 8050.3 & 7231.0 & 8266.0 & 5742.0 & 7079.7 & 7605.2 & 8070.7 & 6361.7 & 7345.8 \\
\hline & Mean & 5526.0 & 5260.2 & 5126.4 & 5304.2 & 4174.8 & 4504.0 & 3768.8 & 4149.2 & 4724.5 & 4610.9 & 4472.5 & 4602.6 \\
\hline 1 & V3N1 & 706.0 & 871.0 & 627.0 & 734.7 & 339.0 & 351.0 & 291.0 & 327.0 & 768.0 & 859.5 & 690.8 & 772.8 \\
\hline 2 & V3N2 & 1241.0 & 1223.0 & 1456.0 & 1306.7 & 888.0 & 900.0 & 1284.0 & 1024.0 & 1198.3 & 1287.7 & 1350.8 & 1278.9 \\
\hline 3 & V3N3 & 2169.0 & 2001.0 & 2259.0 & 2143.0 & 2139.0 & 2137.0 & 1732.0 & 2002.7 & 2025.7 & 1909.2 & 2022.8 & 1985.9 \\
\hline 4 & V3N4 & 2690.0 & 2787.0 & 2390.0 & 2622.3 & 2557.0 & 3013.0 & 2066.0 & 2545.3 & 2550.7 & 2653.2 & 2444.0 & 2549.3 \\
\hline \multirow[t]{2}{*}{5} & V3N5 & 2865.0 & 3084.0 & 2390.0 & 2779.7 & 2782.0 & 3308.0 & 2092.0 & 2727.3 & 2947.7 & 3101.7 & 2578.5 & 2875.9 \\
\hline & Mean & 1934.2 & 1993.2 & 1824.4 & 1917.3 & 1741.0 & 1941.8 & 1493.0 & 1725.3 & 1898.1 & 1962.2 & 1817.4 & 1892.6 \\
\hline 1 & V4N1 & 766.0 & 835.0 & 733.0 & 778.0 & 250.0 & 233.0 & 277.0 & 253.3 & 760.3 & 779.2 & 759.2 & 766.2 \\
\hline 2 & V4N2 & 1797.0 & 1580.0 & 2153.0 & 1843.3 & 1114.0 & 1039.0 & 1629.0 & 1260.7 & 1708.0 & 1548.5 & 2047.0 & 1767.8 \\
\hline 3 & V4N3 & 3013.0 & 2946.0 & 2599.0 & 2852.7 & 2657.0 & 2519.0 & 2210.0 & 2462.0 & 2977.7 & 2753.2 & 2768.3 & 2833.1 \\
\hline 4 & V4N4 & 3323.0 & 3688.0 & 2599.0 & 3203.3 & 2973.0 & 3596.0 & 2315.0 & 2961.3 & 3518.0 & 3774.8 & 2830.8 & 3374.6 \\
\hline \multirow[t]{2}{*}{5} & V4N5 & 3323.0 & 3929.0 & 2599.0 & 3283.7 & 3110.0 & 3810.0 & 2334.0 & 3084.7 & 3677.3 & 4240.5 & 2819.5 & 3579.1 \\
\hline & Mean & 2444.4 & 2595.6 & 2136.6 & 2392.2 & 2020.8 & 2239.4 & 1753.0 & 2004.4 & 2528.3 & 2619.2 & 2245.0 & 2464.2 \\
\hline 1 & V5N1 & 827.0 & 653.0 & 917.0 & 799.0 & 213.0 & 204.0 & 208.0 & 208.3 & 737.2 & 611.0 & 956.8 & 768.3 \\
\hline 2 & V5N2 & 3330.0 & 3702.0 & 2358.0 & 3130.0 & 2142.0 & 1769.0 & 1815.0 & 1908.7 & 2880.2 & 3090.5 & 2182.5 & 2717.7 \\
\hline 3 & V5N3 & 3330.0 & 4390.0 & 2358.0 & 3359.3 & 2961.0 & 3877.0 & 2119.0 & 2985.7 & 3225.3 & 4160.0 & 2276.7 & 3220.7 \\
\hline
\end{tabular}




\begin{tabular}{|c|c|c|c|c|c|c|c|c|c|c|c|c|c|}
\hline & \multicolumn{13}{|c|}{ Continued } \\
\hline 4 & V5N4 & 3330.0 & 4390.0 & 2358.0 & 3359.3 & 2961.0 & 3884.0 & 2119.0 & 2988.0 & 3245.5 & 4328.0 & 2276.7 & 3283.4 \\
\hline \multirow[t]{2}{*}{5} & V5N5 & 3330.0 & 4390.0 & 2358.0 & 3359.3 & 2961.0 & 3884.0 & 2119.0 & 2988.0 & 3262.8 & 4352.0 & 2276.5 & 3297.1 \\
\hline & Mean & 2829.4 & 3505.0 & 2069.8 & 2801.4 & 2247.6 & 2723.6 & 1676.0 & 2215.7 & 2670.2 & 3308.3 & 1993.8 & 2657.4 \\
\hline 1 & N1 & 985.0 & 1027.2 & 994.2 & 1002.1 & 337.4 & 324.6 & 347.0 & 336.3 & 950.8 & 941.7 & 1011.6 & 968.1 \\
\hline 2 & N2 & 2628.0 & 2478.4 & 2788.4 & 2631.6 & 1702.2 & 1498.8 & 1980.8 & 1727.3 & 2411.7 & 2288.9 & 2497.9 & 2399.5 \\
\hline 3 & N3 & 4041.2 & 4062.0 & 3419.4 & 3840.9 & 3381.8 & 3551.0 & 2687.0 & 3206.6 & 3707.2 & 3775.1 & 3171.2 & 3551.2 \\
\hline 4 & N4 & 4514.4 & 5075.0 & 3578.0 & 4389.1 & 3838.4 & 4679.6 & 3048.6 & 3855.5 & 4246.7 & 4836.2 & 3476.3 & 4186.4 \\
\hline 5 & N5 & 4592.2 & 5370.0 & 3578.0 & 4513.4 & 4128.6 & 5053.4 & 3099.2 & 4093.7 & 4547.3 & 5324.0 & 3559.4 & 4476.9 \\
\hline \multicolumn{2}{|c|}{ Overall Mean } & 3352.2 & 3602.5 & 2871.6 & 3275.4 & 2677.7 & 3021.5 & 2232.5 & 2643.9 & 3172.8 & 3433.2 & 2743.3 & 3116.4 \\
\hline
\end{tabular}

Kitumani composite I at $150 \mathrm{~kg} \mathrm{~N} / \mathrm{ha}$ in Santilya, grain yield increase from 6366 $\mathrm{kg} / \mathrm{ha}$ at Spacing two (S2) to $7642 \mathrm{~kg} / \mathrm{ha}$ at spacing one (S1). The results are in accordance with [22] who reported that grain yield increase linearly with increasing plant density provided that there is no limitation of other production factors. [23] reports that grain yield increases until plant densities reach the optimum plant population per unit area.

\subsection{Effect of Nitrogen on Grain yields}

The optimum $\mathrm{N}$ rate (needed to achieve maximum yield) is influenced by factors including soil type, tillage, irrigation, fertilizer timing and method, and crop yield potential. These factors, as well as the interaction of these factors, will vary greatly from one location to another in a given geographic region [24]. The effects of different rates of nitrogen on grain yield of different maize varieties are shown on Table 2. Generally the increasing of the rate of nitrogen application has positive effect on the grain yield of maize in all sites. The grain yield increases until reach the maximum and no further increase in addition of nitrogen. This may be attributed to the significant effect of nitrogen on chlorophyll formation, photosynthesis and assimilate production that resulted in optimum production of yield components which have direct bearing on the final grain yield. This is supported by [25] on the study of the effect of nitrogen in increasing grain yield. In an example of results from Ihumbu farm and Mwazye, for Kitumani Composite I variety at a spacing one (Sp1), the Grain yield increases significantly from $0 \mathrm{~kg} \mathrm{~N} / \mathrm{ha}$ up to the rate of $150 \mathrm{~kg} \mathrm{~N} / \mathrm{ha}$. The following grain yield was obtained at Ihumbu farm, 2058, 3836, 7465 and $8117 \mathrm{~kg} / \mathrm{ha}$ at the rate of $0,50,100$ and $150 \mathrm{~kg} \mathrm{~N} /$ ha respectively. At Mwazye, 576, 2679, 4329 and $4959 \mathrm{~kg} / \mathrm{ha}$ at the rate of $0,50,100$ and $150 \mathrm{~kg} \mathrm{~N} / \mathrm{ha}$ respectively was obtained. At further application of $200 \mathrm{~kg} \mathrm{~N} / \mathrm{ha}$ the increase in Grain yield was insignificantly, $8117 \mathrm{~kg} / \mathrm{ha}$ and $4092 \mathrm{~kg} / \mathrm{ha}$ for Ihumbu farm and Mwazye respectively. This is supported by [26] who reported that the grain yield level off when the supply of nitrogen is beyond the optimum requirement. 


\section{Conclusion}

A different maize variety has been found to vary in grain yielding with respect to nitrogen application and weather condition and soil type. In this study, Maize variety $\mathrm{H} 614$ and Kitumani composite I have been found to respond positively to all factors of production compared to H511, H612 and H626 varieties. The increase in nitrogen application results in increasing grain yield. The optimum amount required differs from one location to another depending on soil type. In most area under the study the rate of $150 \mathrm{~kg} \mathrm{~N} /$ ha was found to be appropriate in increasing grain yield. Spacing has effect on plant population per unit area, reducing spacing from $(90 \mathrm{~cm} \times 30 \mathrm{~cm})$ and leading to $(60 \mathrm{~cm} \times 30 \mathrm{~cm})$ an increase plant population and hence an increasing in grain yield. For the purposes of improving maize production Maize varieties H614 and Kitumani Composite I, Spacing $1(60 \mathrm{~cm} \times 30 \mathrm{~cm})$ and application of nitrogen up to $150 \mathrm{~kg} / \mathrm{ha}$ can be recommended.

\section{Conflicts of Interest}

The authors declare no conflicts of interest regarding the publication of this paper.

\section{References}

[1] Titus, E., Fengying, L. and Cheng, N. (2015) The Role of Agriculture in the Economic Growth and Poverty Reduction in Tanzania. Journal of Economics and Sustainable Development, 6, 154-166.

[2] Mussei, A.N.K. and Shiyumbi, J.K. (1992) Review of the Studies of the Farming Systems of the Southern Highlands of Tanzania, 1970-1990. In: Ekpere, J.A., Rees, D.J., Mbwile, R.P. and Lyimo, N.G., Eds., Proceedings of an International Conference on Agricultural Research, Training and Technology Transfer in the Southern Highlands of Tanzania: Past Achievements and Future Prospects, Mbeya, 5-9 October 1992.

[3] Lyimo, N.G. and Temu, A.E.M. (1992) The Southern Highlands Maize Improvement Programme: Achievements and Strategies for Future Research. In: Ekpere, J.A., Rees, D.J., Mbwile, R.P. and Lyimo, N.G., Eds., Proceedings of an international conference on Agricultural Research, Training and Technology Transfer in the Southern Highlands of Tanzania: Past Achievements and Future Prospects, Mbeya, 5-9 October 1992.

[4] Bisanda, S., Mwangi, W., Verkuij, H., Moshi, A.J. and Anandajayasekeram, P. (1998) Adoption of Maize Production Technologies in the Southern Highlands of Tanzania. International Maize and Wheat Improvement Center (CIMMYT), the United Republic of Tanzania, and the Southern Africa Centre for Cooperation in Agricultural Research (SACCAR).

http://libcatalog.cimmyt.org/download/cim/66324.pdf

[5] Sharratt, B.S. and Mcwilliams, D.A. (2005) Microclimatic and Rooting Characteristics of Narrow-Row vs. Conventional-Row Corn. Journal of Agronomy, 97, 1129-1135. https://doi.org/10.2134/agronj2004.0292

[6] Davi, C.M., Reddy, B.R., Reddy, P.M. and Reddy, S.C.S. (1995) Effects on Nitrogen 
Levels and Plant Density on Yield and Quality of JKHY-1 Cotton. Journal of Agriculture Research, 8, 144-146.

[7] Shen, S. (2002) Contribution of Nitrogen Fertilizer to the Development of Agriculture and Its Loss in China. Acta Ecologica Sinica, 39, 12-25.

[8] Ramos, C. and Varela, M. (1990) Nitrate Leaching in Two Irrigated Fields in the Region of Valencia (Spain). Paris, 335-345.

[9] Wopereis, M.C.S., Tamelokpo, A., Ezui, K., Gnakpenou, D., Fofana, B. and Breman, H. (2006) Mineral Fertilizer Management of Maize on Farmer Differing in Organic Inputs in the West African Savanna. Field Crops Research, 96, 355-362. https://doi.org/10.1016/j.fcr.2005.08.004

[10] Jones, J.W., Hoogenboom, G., Porter, C.H., Boote, K.J., Batchelor, W.D., Hunt, L.A., Wilkens, P.W., Singh, U., Gijsman, A.J. and Ritchie, J.T. (2003) The DSSAT Cropping System Model. European Journal of Agronomy, 18, 235-265. https://doi.org/10.1016/S1161-0301(02)00107-7

[11] Uehara, G. and Tsuji, G.Y. (1998) Overview of IBSNAT. In: Tsuji, G.Y., Hoogenboom, G. and Thornton, P.K., Eds., Understanding Options for Agricultural Production, Springer, Dordrecht, 1-7. https://doi.org/10.1007/978-94-017-3624-4_1

[12] Tsuji, G.Y., Hoogenboom, G. and Thornton, P.K. (1998) Understanding Options for Agricultural Production. Systems Approaches for Sustainable Agricultural Development. Kluwer Academic Publishers, Dordrecht, 400. https://doi.org/10.1007/978-94-017-3624-4

[13] Lazaro, E.A. and Bisanda, S (2005) Local Seed Management Systems for Long-Term Food Security in the Southern Highlands Tanzania Food and Agricultural Organization of the United Nations. Report No. 39.

[14] Bisanda, S., Wilfred, M., Hugo, V., Alfred, J.M. and Ponniah, A. (1998) Adoption of Maize Production Technologies in the Southern Highlands of Tanzania.

[15] Nachtergaele, F., Velthuizen, H.V. and Verelst, V. (2009) Harmonized World Soil Database Version 1.1. FAO/IIASA/ISRIC/ISSCAS/JRC.

[16] Kamprath, E.J., Moll, R.H. and Rodriguez, N. (1973) Effects of Nitrogen Fertilization and Recurrent Selection on Performance of Hybrid Populations of Corn. Agronomy Journal, 74, 955-958.

[17] Uribelarrea, M., Below, F.E. and Moose, S.P. (2004) Grain Composition and Productivity of Maize Hybrids Derived from the Illinois Protein Strains in Response to Variable Nitrogen Supply. Journal of Crop Science, 44, 1593-1600. https://doi.org/10.2135/cropsci2004.1593

[18] Olakojo, S.A. and Iken, J.E. (2001) Yield Performance and Stability of Some Improved Maize Varieties. Moor Journal of Agricultural Research, 2, 21-24.

[19] Khan, M.A., Akbar, S., Ahmad, K. and Baloch, M.S. (1999) Evaluation of Corn Hybrids for Grain Yield. Pakistan Journal of Biological Science, 2, 413-414. https://doi.org/10.3923/pjbs.1999.413.414

[20] Yilmaz, S., Erayman, M., Gozubenli, H. and Can, E. (2008) Twin or Narrow-Row Planting Patterns versus Conventional Planting in Forage Maize Production in the Eastern Mediterranean. Cereal Research Communication, 36, 189-199. https://doi.org/10.1556/CRC.36.2008.1.19

[21] Pinter, L., Alfoldi, Z., Burucs, Z. and Paldi, E. (1994) Feed Value of Forage Maize Hybrids Varying in Tolerance to Plant Density. Agronomy Journal, 86, 799-804. https://doi.org/10.2134/agronj1994.00021962008600050010x 
[22] Anjum, I.J., Ali, C.N., Sahi, E.H., Khan, Z.H. and Jafar, M. (1992) Effect of Plant Population and Fertilizer Application on Growth and Yield of Summer Maize. Journal of Agricultural Research, 30, 453-457.

[23] Tollenaar, M. (1992) Is Low Plant Density a Stress in Maize? Maydica, 37, 305-311.

[24] Gehl, R.J., Schmidt, J.P., Maddux, L.D. and Gordon, W.B. (2005) Corn Yield Response to Nitrogen Rate and Timing in Sandy Irrigated Soils. Agronomy Journal, 97, 1230-1238. https://doi.org/10.2134/agronj2004.0303

[25] Abayomi, Y., George-Arijenja, A. and Kolawole, I.A. (2006) Comparative Leaf Growth and Grain Yield Responses of Hybrid and Open-Pollinated Maize Genotypes to Nitrogen Fertilizer Application. Agrosearch, 8, 13-26.

[26] Marschner, H. (1993) Mineral Nutrition of Higher Plants. Academic Press, Hohenheim, 889 . 